\title{
HIGH AVERAGE CURRENT EFFECTS IN ENERGY RECOVERY LINACS*
}

\author{
L. Merminga ${ }^{\dagger}$, I. E. Campisi, D. R. Douglas, G. A. Krafft, J. Preble, B. C. Yunn \\ Jefferson Lab, Newport News, VA 23606, USA
}

\begin{abstract}
Energy Recovery Linacs (ERLs) can be used as high efficiency driver accelerators to Free Electron Lasers, synchrotron radiation light sources and colliders. Energy recovery has been successfully demonstrated up to $5 \mathrm{~mA}$ of average current in the Jefferson Lab $1.7 \mathrm{~kW}$ IR FEL. Future designs call for much higher average currents of order $100 \mathrm{~mA}$. A number of phenomena can potentially limit the performance of ERLs at these high currents. We review multibunch, multipass instabilities and present experimental data on transverse Beam Breakup and HOM power dissipation obtained at the Jefferson Lab IR FEL. We compare measurements with analytical calculations and simulations.
\end{abstract}

\section{INTRODUCTION}

Superconducting, recirculating linacs with energy recovery can be extremely efficient accelerators for a variety of applications. The efficiency of ERLs increases as the average current is increased. It is therefore important to address the questions: "What is the maximum average current that can be energy recovered, and which effects set this limit?" A number of collective phenomena could potentially limit the maximum current that can be energy recovered. These coherent effects include: a) single bunch effects which are driven by the short time wakefields and limit the charge per bunch, b) multibunch-multipass effects which are driven predominantly by the high- $Q$ superconducting cavities and limit the average current, and finally, c) the power in the HOMs, primarily longitudinal, which depends on the product of bunch charge and average current.

Single bunch effects have been studied systematically in the context of linear colliders and will not be covered here. In this note we focus on multibunch, multipass effects and the HOM power dissipation issue.

We review the common physical mechanism underlying several types of multibunch instabilities in recirculating linacs and present a generalized expression for the threshold current, which is valid for all these types of instabilities, and is derived in the limit of one cavity, one mode and one recirculation. We then describe the experimental investigation of transverse Beam Breakup (BBU) and HOM power dissipation at the Jefferson Lab IR FEL and compare the data with simulations and calculations.

\footnotetext{
*Supported by US DOE Contract No DE-AC05-84-ER40150 †merminga@jlab.org
}

\section{MULTIBUNCH-MULTIPASS INSTABILITIES}

In a recirculating linac, the beam and the cavities form a feedback loop, which closes upon the return of the beam to the same cavity on a subsequent pass. The closure of the feedback loop between beam and cavity can give rise to instabilities at sufficiently high currents. These instabilities include:

1) The transverse BBU instability which results from the interaction of the beam with the cavity's transverse Higher Order Modes (HOMs) [1].

2) The longitudinal BBU instability that can result from the interaction of the beam with longitudinal HOMs [2].

3) The beam-loading type instabilities can arise from fluctuations of the cavity fields in the linac and can cause beam loss on apertures and phase oscillations [3].

\subsection{Single-Cavity Model}

Analytical models for all these types of instabilities have been developed assuming a single cavity, single mode and single recirculation. Perturbative solutions to the models have led to expressions for the threshold current by expanding up to first order in current. The physics of all these effects is fundamentally the same and the threshold current occurs when the power fed into the mode equals the mode power dissipation. Therefore the threshold current depends on the various beam, optical and mode parameters, and is given by a single expression for all three instabilities:

$$
I_{t h}^{(1)}=\frac{-2 p_{r}}{e(R / Q)_{m} Q_{m} k_{m} M_{i j} \sin \left(\omega_{m} t_{r}\right) e^{\omega_{m} t_{r} / 2 Q_{m}}}
$$

Here $p_{r}$ is the momentum of the recirculating beam and $t_{r}$ is the recirculation time. Also $(R / Q)_{m}$ is the shunt impedance of the HOM in units of Ohms, $Q_{m}$ is the HOM quality factor and $k_{m}=\omega_{n} / c$. When $i j=1,2$ or 3,4 and $m$ denotes a transverse HOM, then eq. (1) gives the threshold for the transverse BBU. When $i j=5,6$ and $m$ denotes a longitudinal HOM, then eq. (1) gives the threshold for the longitudinal BBU. When $i j=5,6$ and $m$ denotes the fundamental accelerating mode, then eq. (1) gives the threshold for the beam-loading instability.

Equation (1) is valid only when $M_{i j} \sin (\omega t)<0$. When $M_{i j} \sin (\omega t)>0$, the first order expansion of the analytic solution fails to yield a physically meaningful threshold, and a higher order solution must be sought. For the case of the transverse BBU instability, one can show that, when $M_{12} \sin (\omega t)>0$, a second-order expansion in current yields a positive threshold given by: 


$$
\begin{aligned}
& I_{t h}^{(2)}=\frac{4 p_{r} c}{e(R / Q)_{m} Q_{m} k_{m} M_{12} \sin \left(\omega_{m} t_{r}\right)(\sqrt{\Lambda}-1) e^{\omega_{m} t_{r} / 2 Q_{m}}} \\
& \Lambda \equiv 1+\frac{2 \omega_{m} t_{0}}{Q_{m}} \cot \left(\omega_{m} t_{0}\right) \cot \left(\omega_{m} t_{r}\right)
\end{aligned}
$$$$
-\frac{4 \omega_{m} t_{r}}{Q_{m}} \cot \left(\omega_{m} t_{r}\right) \cot \left(2 \omega_{m} t_{r}\right)
$$

Typically the limiting instability in recirculating linacs is the transverse BBU instability. We experimentally investigated transverse BBU in the Jefferson Lab IRFEL and we are in the process of analyzing the data. We describe the experiment and present preliminary results from the data analysis next.

\subsection{Transverse BBU Experiment}

The experiment consisted of measurements of the beam transfer functions in the recirculating mode. Although these measurements were performed at beam currents lower than the threshold current, yet they lead to clear estimates of the instability threshold. A $50 \mathrm{Ohm}$ stripline BPM was used to impart transverse momentum to the beam with the modulating frequency of the HOMs under study. A 300 W TWT amplifier was driven by a vector network analyzer at the proper frequency, sweeping the frequency across the HOM frequency. The power from the TWT was split with a $90^{\circ}$ coaxial hybrid, and the two divided ports phase shifted so that the two vertical ports of the BPM would be powered $180^{\circ}$ out of phase, to increase the modulation without increasing the power through the individual BPM feedthroughs. Power levels of the order of $30 \mathrm{~W}$ per port were used during the tests. The signal from the cavities under test was fed back to the network analyzer's input port to complete the $S_{21}$ measurement. Fig. 1 displays a frequency scan of the HOM resonance at $1887 \mathrm{MHz}$ at various currents, from 0 to $4 \mathrm{~mA}$. Several modes were excited in two different cavities.

Table 1: BBU Experimental Settings

\begin{tabular}{|c|c|c|c|c|c|c|}
\hline $\begin{array}{c}\text { HOM } \\
\text { freq. }\end{array}$ & $\begin{array}{c}\mathrm{R} / \mathrm{Q} \\
\text { Meas. }\end{array}$ & $\begin{array}{c}\mathrm{Q} \\
\text { Meas. }\end{array}$ & $\mathrm{E}_{\text {beam }}$ & $\begin{array}{c}\text { Opt. } \\
\text { Set. }\end{array}$ & $\mathrm{I}_{\text {th }}{ }^{(1)}$ & $\mathrm{I}_{\text {th }}{ }^{(2)}$ \\
\hline $\mathrm{MHz}$ & $\Omega$ & $\mathrm{x} 10^{5}$ & $\mathrm{MeV}$ & & $\mathrm{mA}$ & $\mathrm{mA}$ \\
\hline 1730 & 0.08 & 380 & 48 & 0 & 16.0 & \\
\hline 1730 & 0.08 & 380 & 37 & 1 & 18.4 & \\
\hline 1895 & 22.02 & 1.6 & 48 & 0 & $<0$ & 21.4 \\
\hline 1895 & 22.02 & 1.6 & 37 & 1 & $<0$ & 15.6 \\
\hline 1895 & 22.02 & 1.6 & 37 & 0 & $<0$ & $<0$ \\
\hline 1818 & 13.74 & 0.45 & 37 & 2 & $<0$ & 15.0 \\
\hline 1818 & 13.74 & 0.45 & 37 & 3 & $<0$ & 6.9 \\
\hline 1887 & 22.21 & 4.0 & 37 & 3 & 12.5 & \\
\hline 1887 & 22.21 & 4.0 & 37 & 4 & $<0$ & 11.3 \\
\hline 1887 & 22.21 & 4.0 & 37 & 2 & 32.0 & \\
\hline 1887 & 22.21 & 4.0 & 37 & 3 & 16.4 & \\
\hline
\end{tabular}

Table 1 presents the various conditions under which data were recorded. Modes $1730 \mathrm{MHz}$ and $1895 \mathrm{MHz}$ exist in cavity 4, while modes 1818 and $1887 \mathrm{MHz}$ exist in cavity 5 . The $R / Q$ and $Q$ values have been determined experimentally [4,5]. The fourth column displays the recirculated beam energy. Most data were recorded at 37 $\mathrm{MeV}$ for increased sensitivity. The fifth column displays the particular optics setting used in the recirculator. Setting 0 corresponds to the nominal configuration used during lasing.

\subsection{BBU Data Analysis}

Data analysis of the BBU instability requires that the measured signal $V_{c}(\omega)$ and drive signal $V_{k}(\omega)$ be expressed in terms of the threshold current of the instability. Following the analysis described in [6] one arrives at:

$$
\frac{V_{c}(\omega)}{V_{k}(\omega)}=\alpha_{c} \frac{W(\omega)}{\alpha_{k}}\left(\frac{I_{0}}{1-I_{0} / I_{t h}}\right)
$$

where $S_{2 I}(\omega) \equiv V_{c}(\omega) / V_{k}(\omega)$ and $I_{t h}$ denotes the threshold current. The instability denominator $D(\omega)=1-I_{0} / I_{t h}$ is zero at the threshold current. Taking the modulus and the logarithm of both sides yields,

$$
\log \left|S_{21}(\omega)\right|=a_{0}+\log \left(I_{0}\right)-\log \left(1-a_{1} I_{0}\right)
$$

The network analyzer was set up to measure the amplitude of $S_{21}(\omega)$ in $\mathrm{dB}$ as a function of average current $I_{0}$. Parameters $a_{0}$ and $a_{1}$ can be fitted to the data. $a_{0}$ contains information about the HOM impedance and $a_{l}=1 / I_{t h}$. This nonlinear fit in parameter $a_{l}$ yields the threshold currents displayed in Table 1, column 6. For a number of datasets however, this type of fit was unable to produce positive thresholds. There appears to be a strong correlation between the sets for which the fit failed and the product of $M_{34} \sin (\omega t)$ being positive. The expansion to second order in current which yielded eq. (2) was then used, and the data were fitted to:

$\log \left|S_{21}(\omega)\right|=a_{0}+\log \left(I_{0}\right)-\log \left[\left(1-a_{1} I_{0}\right)\left(1+a_{2} I_{0}\right)\right]$ where both parameters $a_{1}$ and $a_{2}$ were constrained to be positive. The last column of Table 1 displays the values of the threshold currents obtained by this second order fit. There is still one data set for which both fits yield negative currents.

Note that under all the different optics configurations used, different mode excitations and different beam energies, the threshold current varies between approximately $7 \mathrm{~mA}$ and $32 \mathrm{~mA}$. Furthermore, the threshold current for the nominal configuration is either $16 \mathrm{~mA}$ or $21 \mathrm{~mA}$, depending on the mode excited. Simulations with the numerical code TDBBU [7] predict a threshold current for the nominal FEL configuration of $27 \mathrm{~mA}$. The small discrepancy is possible because the calculated TDBBU threshold was obtained without taking into account the effect of $\mathrm{rf}$ focusing. Detailed difference orbit data have been recorded that will allow us to quantitatively characterize the optics for each of the configurations used in the experiment. When the analysis is complete, detailed comparisons between TDBBU and the data will take place, and a more precise statement on 
the agreement between TDBBU and experimental data could be made.

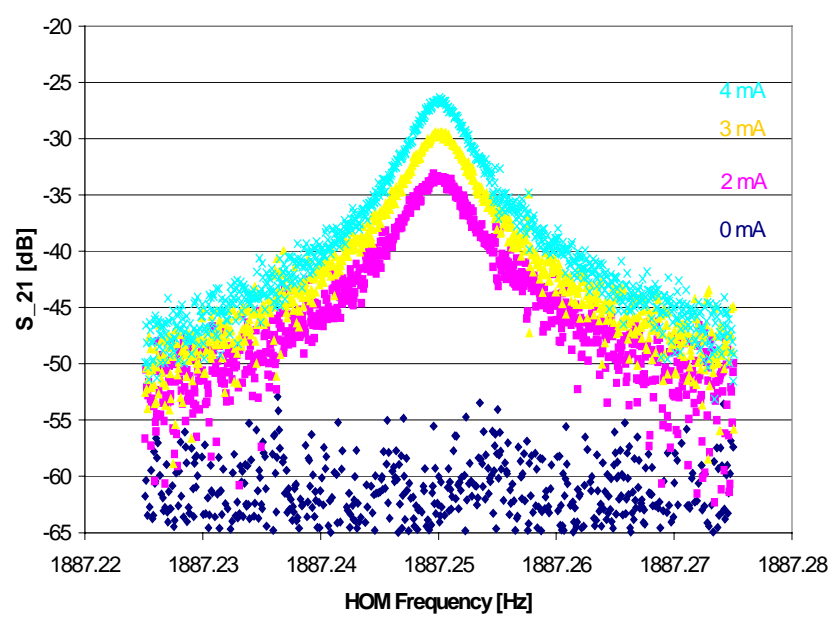

Figure 1: Excitation of the $1887 \mathrm{MHz}$ mode at various currents.

\section{HOM POWER DISSIPATION}

The HOM power transferred to the loads was measured in the energy recovery mode in the JLab FEL as function of bunch charge and bunch repetition frequency, at the nominal bunch length setting used during lasing. Temperature diodes were placed at various locations around the cryomodule's cavities, including one on each HOM load (there are two HOM loads per cavity). The diode readings were calibrated with a known amount of heater power. Data were recorded first with beam off and then with beam on in steps of $20 \mathrm{pC}$ per bunch, up to 80 $\mathrm{pC}$, at four different bunch repetition frequencies: 9,18 , 37 and $75 \mathrm{MHz}$, with each frequency being precisely a factor of two higher than the previous one. Figure 2 displays power vs. bunch charge, for 18, 37 and $75 \mathrm{MHz}$. The two figures correspond to the two HOM loads of a cavity. Since the power dissipated in the HOMs is given by $P_{\text {HOM }}=2 k_{\|} Q^{2} f_{\text {rep }}$ (the factor of 2 accounts for the two beams in the linac) where $Q$ is the charge per bunch, $f_{\text {rep }}$ is the bunch repetition frequency and $k_{\|}$is the loss factor of the CEBAF 5-cell cavity, the data were fitted to functions of the form: $a Q^{2}, 2 a Q^{2}$ and $4 a Q^{2}$ for the 18,37 and $75 \mathrm{MHz}$ respectively. The loss factor derived from these least-square fits is $9.4 \mathrm{~V} / \mathrm{pC}$. At the nominal bunch length, the calculated loss factor is $11 \mathrm{~V} / \mathrm{pC}$. We do not presently have an explanation for the asymmetry between the power in the two loads. More measurements are planned to verify and understand the asymmetry. Finally, at the present time, no statement can be made on the amount of power dissipated in the cryogenic environment. In order to quantify this statement one would need to measure the helium mass flow, which is not possible with the present setup.

\section{CONCLUSIONS}

High average current effects in recirculating, energy recovering linacs are theoretically well-understood. Experimental verification of simulation codes and models is being pursued in the Jefferson Lab IR FEL and analysis is in progress. To date, quantitative agreement has been demonstrated between simulation and experiment for transverse BBU and HOM power dissipation. Greater capabilities for experimental verification of the models are offered with the JLab FEL Upgrade at $10 \mathrm{~mA}$ average current and the Cornell/Jefferson Lab ERL Prototype at $100 \mathrm{~mA}$ average current, both described in these proceedings by D. Douglas and I. Bazarov respectively.
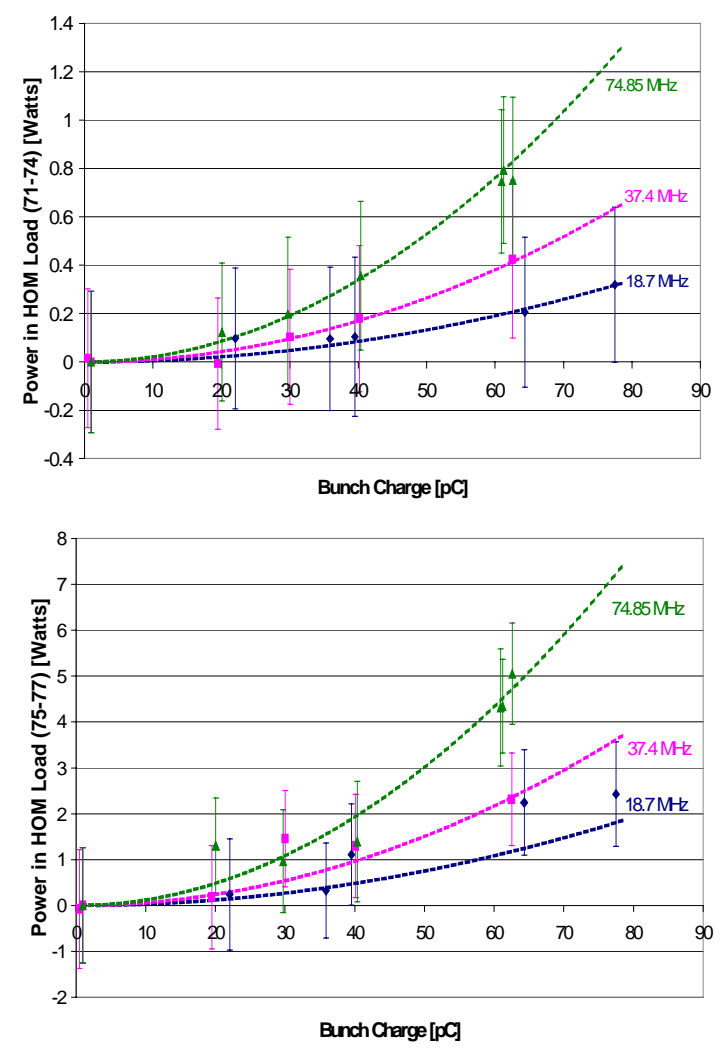

Figure 2: HOM power measured in the two HOM loads of the CEBAF 5-cell cavities vs. bunch charge for 3 different bunch repetition rates.

\section{REFERENCES}

[1] J. Bisognano and Gluckstern, Proc. PAC 1078 (1987)

[2] J. Bisognano and M. Fripp, Proc. LINAC Conf. (1988)

[3] L. Merminga, et al., NIM A 375, ABS39-41 (1996) and NIM A 429: 58-64 (1999)

[4] J. Amato, Cornell Note SRF-831002-EXA (1983)

[5] Campisi and Merminga, JLab Note TN-98-011 (1989)

[6] N. Sereno, Ph.D. Thesis, U. of Illinois (1994)

[7] G. Krafft and Bisognano, Proc. PAC, pg.1356 (1887) 